\title{
Prognostic and pathogenic role of CXC Motif Ligand 16 in sepsis
}

\author{
Jiaxi $\mathrm{Li}^{1}$, Lili Huang ${ }^{1}$, Yi Liu ${ }^{2}$, Yi Gong${ }^{1}$, and Ju Cao ${ }^{1}$ \\ ${ }^{1}$ The First Affiliated Hospital of Chongqing Medical University \\ ${ }^{2}$ Chongqing Medical University Affiliated Second Hospital
}

November 4, 2020

\begin{abstract}
Chemokine CXC motif ligand 16 (CXCL16) is a multifaceted chemokine that has been shown to participate in a variety of inflammatory diseases. The role of CXCL16 in the immunopathology of sepsis remains unidentified. In this study, human patients with sepsis and healthy controls were used to obtain blood for in vitro studies, and female C57BL/6J mice were taken for in vivo studies. The effects of recombinant CXCL16 protein or anti-CXCL16 monoclonal antibody on sepsis were evaluated in a murine model of cecal ligation and puncture (CLP)-induced polymicrobial sepsis. On admission, human patients with sepsis had significantly higher soluble levels of serum CXCL16 than healthy controls. Soluble CXCL16 remained significantly elevated in septic patients from day 0 to 7 . Admission levels of soluble CXCL16 were positively correlated disease severity and the serum levels of other inflammatory cytokines and chemokines. Furthermore, nonsurvivors displayed significantly higher admission levels of soluble CXCL16 compared with survivors of septic patients. Soluble CXCL16 levels revealed significant prognostic value for 28-day mortality, and CXCL16 was found to be an independent predictor of 28-day mortality in septic patients. In CLP-induced nonsevere sepsis, administration with recombinant CXCL16 increased mortality and tissue injury. Conversely, neutralizing CXCL16 by anti-CXCL16 monoclonal antibody decreased mortality and tissue injury in CLP-induced severe sepsis. However, CXCL16 did not affect the ability of these mice to clear bacteria in CLP. Taken together, CXCL16 could be linked to sepsis not only as a new marker of prognosis, but also as a potential target for therapeutic intervention.
\end{abstract}

\section{Prognostic and pathogenic role of CXC Motif Ligand 16 in sepsis}

Jiaxi Li ${ }^{1 \#}$, Lili Huang ${ }^{1 \#}$, Yi Liu ${ }^{2}$, Yi Gong ${ }^{3}, \mathrm{Ju} \mathrm{Cao}^{1 *}$.

${ }^{1}$ The Department of Laboratory Medicine, The First Affiliated Hospital of Chongqing Medical University, Chongqing, China;

${ }^{2}$ Department of Intensive care unit, The Second Affiliated Hospital of Chongqing Medical University, Chongqing, China;

${ }^{3}$ Department of Blood Transfusion, The First Affiliated Hospital of Chongqing Medical University, Chongqing, China.

\# These authors contributed equally to this work.

*Correspondence: Professor Ju Cao, Department of Laboratory Medicine, The First Affiliated Hospital of Chongqing Medical University, $1^{\#}$ Youyi Road, District Yuzhong, Chongqing, China, Telephone Number: +86-23-89012513, Fax: +86-23-89012513, Email: caoju723@163.com.

Running title: The role of CXCL16 in sepsis

Key words: CXCL16; sepsis; inflammation; infection; prognosis

\section{Summary}


Chemokine CXC motif ligand 16 (CXCL16) is a multifaceted chemokine that has been shown to participate in a variety of inflammatory diseases. The role of CXCL16 in the immunopathology of sepsis remains unidentified. In this study, human patients with sepsis and healthy controls were used to obtain blood for in vitro studies, and female C57BL/6J mice were taken for in vivo studies. The effects of recombinant CXCL16 protein or anti-CXCL16 monoclonal antibody on sepsis were evaluated in a murine model of cecal ligation and puncture (CLP)-induced polymicrobial sepsis. On admission, human patients with sepsis had significantly higher soluble levels of serum CXCL16 than healthy controls. Soluble CXCL16 remained significantly elevated in septic patients from day 0 to 7 . Admission levels of soluble CXCL16 were positively correlated disease severity and the serum levels of other inflammatory cytokines and chemokines. Furthermore, nonsurvivors displayed significantly higher admission levels of soluble CXCL16 compared with survivors of septic patients. Soluble CXCL16 levels revealed significant prognostic value for 28-day mortality, and CXCL16 was found to be an independent predictor of 28-day mortality in septic patients. In CLP-induced nonsevere sepsis, administration with recombinant CXCL16 increased mortality and tissue injury. Conversely, neutralizing CXCL16 by anti-CXCL16 monoclonal antibody decreased mortality and tissue injury in CLP-induced severe sepsis. However, CXCL16 did not affect the ability of these mice to clear bacteria in CLP. Taken together, CXCL16 could be linked to sepsis not only as a new marker of prognosis, but also as a potential target for therapeutic intervention.

Key words: CXCL16; sepsis; inflammation; infection; prognosis

\section{Introduction}

Sepsis is defined as a life-threatening organ dysfunction that is caused by a dysregulated host response to infection [1]. Globally, it is estimated that there are 31.5 million sepsis and 19.4 million severe sepsis cases per year, and up to 5.3 million deaths annually [2]. In 2017, the world health organization (WHO) declared sepsis as a disease of global health priority, needing special attention due to its high prevalence and mortality worldwide [3]. In sepsis, the host immune response that is triggered by microbial infection fails to return to normal homeostasis, involving both sustained excessive inflammation and immune suppression [4]. Identification of host immune regulators participating in disturbing host immune response may point toward novel therapeutic leads in sepsis.

The CXC chemokine ligand 16 (CXCL16) is an unusual chemokine that exists in both transmembrane and soluble forms [5]. The protein structure of CXCL16 comprises a small C-terminal intracellular domain and an extracellular N-terminal chemokine domain bound to the transmembrane region through a heavily glycosylated mucin-like stalk [6]. CXCL16 is mainly produced by macrophages and dendritic cell, but is also produced by lymphocytes, fibroblasts, smooth muscle cells, and endothelial cells [7]. Surface-expressed CXCL16 can mediate adhesion to cells expressing its specific receptor CXCR6, while the soluble form of CXCL16 shed from the surface can act as a chemotactic gradient for leukocytes expressing CXCR6 [8]. CXCL16 expression has been related to a variety of human inflammatory diseases including rheumatoid arthritis [9], systemic lupus erythematosus (SLE) [10], lung cancer [11], thyroid cancer [12], hepatocellular carcinoma [13], lung injury [14], Salmonella enterica serovar Enteritidis [7], and human immunodeficiency virus (HIV) infection [15]. Besides, several studies support usage of soluble CXCL16 as a biomarker of Inflammation, atherosclerosis, and acute coronary syndromes in humans [16-18]. However, little is known about the expression and function of CXCL16 in sepsis.

In order to investigate the potential role of CXCL16 in sepsis, we accompanied human clinical studies with in vivo mice experiments. Firstly, we analyzed the extent of soluble CXCL16 over time in the patients with sepsis and its correlation with disease outcome. Furthermore, we examined the impact of CXCL16 on host immune responses, tissue injury, and mortality in the cecal ligation and puncture (CLP) model of polymicrobial sepsis by administration of recombinant CXCL16 protein or neutralizing anti-CXCL16 antibody. We here reported that CXCL16 might be an important inflammatory mediator in the pathogenesis of sepsis.

\section{Materials and Methods}

\section{Septic patients and healthy controls}


55 septic patients who met the clinical criteria for sepsis-3 [1] were recruited on admission to the Intensive Care Unit (ICU) of The Second Affiliated Hospital of Chongqing Medical University between October 2017 and March 2019. Clinical and demographic characteristics of patients were collected, including age, gender, co-morbidities, white blood cells (WBC), procalcitonin (PCT) and c-reaction protein (CRP), Acute Physiology and Chronic Health Evaluation (APCHE) II score and Sequential [Sepsis-related] Organ Failure Assessment (SOFA) score at $24 \mathrm{~h}$ on admission. During the follow-up, other parameters were also recorded, such as microbiological findings, ventilation, use of vasopressors, use of hydrocortisone, renal replacement therapy, length of ICU stay, and mortality during the 28-day study period. Patients with pregnancy or breast-feeding, malignancy, organ transplantation, human immunodeficiency virus infection (HIV) infection, viral hepatitis, autoimmune diseases, and the use of immunosuppressive agents in the past 8 weeks were excluded. Peripheral blood was collected at the time of patient admission, and serum was then isolated and stored at -80 . 30 age- and sex- matched healthy control blood samples were taken from healthy donors with no medical problems in the medical examination center. Human studies were reviewed and approved by the Clinical Research Ethics Committee of Chongqing Medical University (Registration number: LLZM-20120016), and informed consent was obtained from all participants according to the Declaration of Helsinki.

\section{Sepsis animal model}

CLP was used to establish a model of polymicrobial sepsis as described in our previous studies [19-21]. Briefly, female C57BL/6 mice aged 6-8 weeks were firstly anesthetized intraperitoneally (i.p.) with a mixture of xylazine $(4.5 \mathrm{mg} / \mathrm{kg})$ and ketamine $(90 \mathrm{mg} / \mathrm{kg})$, and the cecum was then exposed, ligatured at its external third, and punctured through with a 24 -gauge needle (nonsevere CLP, $60-80 \%$ survival) or a 21-gauge needle (severe CLP, 20-40\% survival). The cecum was then returned to the peritoneal cavity, and incisions were closed. Sham-operated (control) animals underwent identical laparatomy, and the cecum was exposed but not ligated or punctured. Mice received saline ( $5 \mathrm{ml}$ per $100 \mathrm{~g}$ body weight) subcutaneously for resuscitation. Survival was monitored twice daily for $14 \mathrm{~d}$. All animal experiments were approved by the local Animal Care and Use Committee.

\section{Administration with recombinant mouse CXCL16}

Recombinant murine CXCL16 protein (R\&D systems, Catalog \# 503-CX-025) was administered i.p. into septic C57BL/6 mice immediately after nonsevere CLP. Phosphate buffer saline (PBS) was used as control vehicle.

\section{Antibody-mediated blockade of CXCL16}

To neutralize the activity of CXCL16 in severe CLP, rat anti-mouse CXCL16 neutralizing monoclonal antibody (R\&D systems, monoclonal rat IgG2A Clone \# 142417) was used. The neutralization ability of antiCXCL16 monoclonal antibody has been confirmed by its capacity to neutralize CXCL16-induced chemotaxis in the BaF3 mouse pro-B cell line transfected with mouse CXCR6. The Neutralization Dose (ND50) is typically $0.15-0.6 \mu \mathrm{g} / \mathrm{mL}$ in the presence of $7.5 \mathrm{ng} / \mathrm{mL}$ recombinant mouse CXCL16 chemokine domain (R\&D systems, Catalog \# 503-CX-025)). In mouse studies, $10 \mu \mathrm{g}$ of anti-mouse CXCL16 neutralizing monoclonal antibody was injected i.p. in $50 \mu \mathrm{l}$ of PBS 2 hours after CLP, and rat IgG2A control antibody was used as control.

\section{Measurement of cytokines and chemokines}

The concentrations of CXCL16 and KC (IL-8 murine homolog) were quantified using commercially available enzyme-linked immunosorbent assay (ELISA) kits (R\&D Systems). The levels of other inflammatory cytokines and chemokines including IL-6, IL-8, IL-27, and TNF- $\alpha$ were assessed with commercially available ELISA kits (Biolegend). In order to quantify cytokines and chemokines in peritoneal lavage fluid (PLF), 5 $\mathrm{ml}$ of cold PBS was injected into the peritoneal cavity. PLF was then collected for further analysis.

\section{Serum biochemistry}

Blood was collected from C57BL/6 mice in tubes with heparin after cardiac puncture. The levels of alanine 
aminotransferase (ALT), aspartate transaminase (AST), lactate dehydrogenase (LDH), and creatinine were quantified by spectrophotometric analyzer (modular DP; Roche-Hitachi, Echevarne Laboratories).

\section{Differential leukocyte counts}

Peritoneal cell suspension in PLF was pelleted and resuspended. Cell viability was firstly characterized by Trypan blue exclusion assay, and cell numbers were quantified in a hematology analyzer. Cytospin slides were finally prepared and stained by Wright-Giemsa stain.

\section{Histopathology}

The lungs, livers, and kidneys from CLP and sham mice were collected, fixed, sectioned, and stained with hematoxylin and eosin (HE) at the indicated times. The following parameters including bronchitis, edema, interstitial inflammation, intra-alveolar inflammation, pleuritis, endothelialitis, and the percentage of the lung surface demonstrating confluent inflammatory infiltrate were used to score lung inflammation and damage as described in our previous studies. The pathology scores of livers and kidneys were determined according to the following variables: number of thrombi, number of (micro) abscesses, presence and degree of inflammation, and presence and degree of necrosis.

\section{Calculation of bacterial colony-forming unit (CFU)}

Serial dilutions of whole blood or PLF of C57BL/6 mice after CLP or sham surgery were plated on blood agar plates overnight at $37^{\circ} \mathrm{C}$. CFUs were then calculated.

\section{Statistical analysis}

Data are expressed as scatter dot plots with medians or as box and whisker plots showing the smallest observation, lower quartile, median, upper quartile, and largest observation or as medians with interquartile ranges. Mann-Whitney $U$ test or Kruskal-Wallis test followed by Dunn's multiple comparisons post test was performed for statistical analysis. Non-parametric Spearman's Rank Correlation Coefficient was used to analyze correlations. To determine the discriminative power of human soluble CXCL16 for 28-day mortality, receiver-operating characteristic (ROC) curves were constructed and the area under the curve (AUC) was determined with its $95 \%$ confidence interval (CI). For comparisons of AUCs, the Z-test formula was used. Binary logistic regression analysis was performed to determine the independent predictors of 28-day mortality. For survival studies, Kaplan-Meier analyses followed by log-rank tests were performed. All analyses were done with GraphPad Prism version 5.01 (GraphPad Software, San Diego, CA). $p$ values less than 0.05 were considered statistically significant.

\section{Results}

\section{Patient characteristics}

55 patients with sepsis were recruited in this study. The basic demographics and clinical characteristics of these patients are described in supplementary Table 1 . The 28 -day mortality was $34.5 \%$ in the patients with sepsis $(\mathrm{n}=19)$. The identified pathogens related to the patients with sepsis are described in supplementary Table 2. Nonsurvivors had significantly greater APCHEII and SOFA scores on ICU admission than survivors. The concentrations of lactate, PCT, and CRP were also significantly higher in nonsurvivors than in survivors (Supplementary Table 3).

\section{The concentrations of soluble CXCL16 were increased in the patients with sepsis}

In the serum of 55 septic patients, admission levels of soluble CXCL16 were significantly increased than those of healthy individuals (Figure 1A). Soluble CXCL16 levels were still significantly elevated on day 7 compared with healthy individuals (Figure 1B). Soluble CXCL16 concentrations were significantly higher in septic shock patients compared to septic patients without shock (Figure 1C), and non-surviving patients with sepsis displayed significantly increased CXCL16 levels compared to survivors (Figure 1D). However, there was no statistical difference in circluating CXCL16 concentrations between bacteremia patients and non-bacteremic patients on ICU admission (Supplementary Figure 1). 


\section{Admission levels of CXCL16 correlated with disease severity in the patients with sepsis}

Serum soluble CXCL16 levels were significantly correlated with SOFA scores on day of ICU admission (Figure 2A). Moreover, admission levels of soluble CXCL16 correlated with WBC numbers (Figure 2B) and the concentrations of CRP (Figure 2C).

To investigate whether soluble CXCL16 levels are correlated with other cytokine levels in the serum of patients with sepsis, we also assessed the serum concentrations of IL-6, IL-8, IL-27 and TNF- $\alpha$. Soluble CXCL16 levels and levels of cytokines including IL-6, IL-8, IL-27 and TNF- $\alpha$ were significantly correlated at day of ICU admission (Supplementary Table 4).

The clinical role of CXCL16 in predicting 28-day mortality of septic patients

The AUC for prediction of 28-day mortality were calculated for CXCL16, CRP, PCT and SOFA scores. The AUC for CXCL16 on day of ICU admission was 0.717 ([95\% CI] $0.497-0.939, p=0.047$, Figure 3), higher than the AUC for PCT (AUC = 0.617, $[95 \% \mathrm{CI}]$ 0.429-0.805, $p=0.231$ ), and CRP (AUC $=0.521,[95 \%$ CI] $0.338-0.705, p=0.819)$, but lower than the AUC for SOFA $(\mathrm{AUC}=0.849,[95 \% \mathrm{CI}] 0.715-0.982, p<$ 0.001). Furthermore, both CXCL16 $(B=0.007, \mathrm{OR}=1.004, p=0.039)$ and SOFA score $(B=0.090, \mathrm{OR}=$ $1.093, p=0$ ) on day of ICU admission were found to be independent predictors of 28-day mortality in the patients with sepsis, but PCT $(B=0.013$, OR $=1.011, p=0.074)$ and $\mathrm{CRP}(B=0.004$, OR $=1.006, p=$ 0.304) were not (Table 1 ).

\section{Administration of recombinant murine CXCL16 protein worsened sepsis-induced mortality}

We then studied the functional role of CXCL16 in the model of CLP-induced polymicrobial sepsis. The protein concentrations of CXCL16 were substantially up-regulated in the lung, PLF and blood after CLP (Supplementary Figure 2). The effects of increasing doses $(0.5-1.0 \mu \mathrm{g})$ of recombinant murine CXCL16 on nonsevere sepsis were firstly assessed in a sublethal model of CLP. We found that treatment with recombinant murine CXCL16 in the absence of sepsis did not affect survival at doses up $1.0 \mu \mathrm{g}$ in sham control mice. However, supplementation of recombinant murine CXCL16 $(0.5-1.0 \mu \mathrm{g})$ significantly decreased mice survival compared with the PBS-injected septic mice (Figure 4A). Our subsequent experiments were thus performed in CLP mice using recombinant murine CXCL16 $(0.5 \mu \mathrm{g})$ immediately after nonsevere CLP.The decrease in survival of septic mice treated with recombinant CXCL16 was related to increased tissue inflammation and organ injury. At 24 hours after nonsevere CLP, inflammation of lung, liver, and kidney was increased (Figure 4B), which was reflected by significantly higher pathology scores in mice treated with recombinant CXCL16 as compared with PBS-treated mice (Figure 4C). Moreover, supplementation of recombinant murine CXCL16 to septic mice significantly increased serum concentrations of ALT, AST, LDH, and creatinine, suggesting a detrimental enhancement of liver, kidney, and cardiac injury (Figure 4D). The decreased survival rate in the mice treated with recombinant murine CXCL16 could not be explained by impaired bacterial clearance, because there was no statistical difference in local or systemic bacterial CFU levels in the septic mice treated with recombinant CXCL16 compared with mice treated with PBS control (Supplementary Figure 3).

Administration of recombinant murine CXCL16 augmented inflammation after experimental sepsis induction

The leukocyte numbers in PLF from septic mice treated with recombinant murine CXCL16 were significantly greater than those of PBS-treated septic mice (Figure 5A), which comprised mainly neutrophils, monocytes/macrophages, and lymphocytes. Furthermore, CXCL16 administration enhanced the cytokine and chemokine response in nonsevere CLP, which led to a significant elevation of IL-6 TNF- $\alpha$, KC and IL-27 in PLF and blood (Figure 5B).

\section{Anti-CXCL16 antibody protected mice from severe sepsis}

Because the administration of recombinant murine CXCL16 worsened sepsis-induced mortality, we performed the reverse experiment and investigated the effects of blocking CXCL16 using a mouse anti-CXCL16 antibody in severe CLP. The survival rate in septic mice treated with anti-CXCL16 blocking antibodies was 
significantly greater than that in the IgG-treated control group (Figure 6A). Lung, liver, and kidney inflammation was significantly decreased in septic mice treated with anti-CXCL16 antibody as compared with mice treated with isotypic IgG (Figure 6B). Blockade of CXCL16 significantly decreased the pathology scores for lungs, livers, and kidneys after severe sepsis (Figure 6C). Furthermore, serum levels of ALT, AST, LDH and creatinine were significantly down-regulated in septic mice treated with anti-CXCL16 antibody at $24 \mathrm{~h}$ after CLP (Figure 6D). Remarkably, treatment with anti-CXCL16 antibody did not affect the bacterial clearance in mice after severe CLP, and mice treated with anti-CXCL16 antibody had similar bacterial loads from peritoneum and blood when compared with mice treated with IgG control after severe CLP (Supplementary Figure 4).

\section{Discussion}

Over the past years, the chemokine has emerged to be an important inflammatory mediator of various human diseases. In the present report, we identified CXCL16 to be centrally involved in the pathogenesis of sepsis. We made the following key observations: I. Sepsis patients had a marked and sustained elevation of soluble CXCL16. II. Admission levels of soluble CXCL16 levels correlated with disease severity and mortality in human patients with sepsis. III. CXCL16 was found to be an independent predictor of 28-day mortality in the patients with sepsis on day of ICU admission. IV. CXCL16 supplementation in the presence of sepsis could worsen outcome of CLP-induced nonsevere sepsis. V. Neutralization of the CXCL16 activity with antibody against CXCL16 could produce substantial survival benefit in CLP-induced severe sepsis.

It has been suggested that soluble CXCL16 could serve as a reliable marker of inflammation. ${ }^{16}$ Indeed, soluble levels of serum CXCL16 were correlated significantly with the disease activity of patients with inflammatory bowel disease (IBD) [22]. Consistent with this pro-inflammatory profile, soluble CXCL16 levels were independently associated with coronary atherosclerosis, particularly acute coronary syndromes. ${ }^{16}$ In patients with an acute coronary syndrome, CXCL16 levels obtained within 24 hours of admission are associated with long-term mortality after adjustment for other risk factors [23]. Furthermore, admission level of CXCL16 is independently related to adverse clinical outcomes in patients with an acute coronary syndrome, mainly driven by an association to cardiovascular death [18]. Therefore, CXCL16 measurement may enhance risk stratification in patients with acute coronary syndrome. However, to our knowledge CXCL16 has never been evaluated in the patients with sepsis. This study revealed dramatically increased soluble CXCL16 levels in septic patients on day of ICU admission as compared to healthy controls. Further, we observed that admission levels of soluble CXCL16 significantly differed between survivors and non-survivors in the patients with sepsis. Moreover, admission levels of CXCL16 were positively correlated with SOFA score and different laboratory outcome parameters such as WBC, CRP, IL-6, IL-8, IL-27 and TNF- $\alpha$. We therefore tested for an additive prognostic value of soluble CXCL16 levels in septic patients. We demonstrated, for the first time, the association between increased admission levels of soluble CXCL16 and higher risk of death in the patients with sepsis. Although the AUC of CXCL16 was lower than that of SOFA score in predicting 28-day mortality, it was superior to PCT and CRP for predicting 28-day mortality in septic patients. In the logistic regression analysis, CXCL16 was found to be independent predictors of 28-day mortality in septic patients. These findings suggest that CXCL16 might be added to the list of risk markers that could identify a group of septic patients presenting with higher risk of mortality beyond that of other risk indicators, such as soluble ST2 [24], presepsin [25], soluble IL-7 receptor [26], and apoptosis inhibitor of macrophage (AIM) $[27]$.

CXCL16 up-regulation has been shown to participate in the inflammatory response elicited during bacterial and viral infections, which was associated with either beneficial or detrimental effects [6,7]. To further investigate the role of CXCL16 in the pathogenesis of sepsis, we established a clinically relevant murine model of sepsis induced by CLP. Consequently, we found that supplementation with recombinant CXCL16 decreased survival and increased tissue inflammation and tissue injury in mice after non-severe CLP, while neutralization of CXCL16 resulted in substantial survival benefit after severe-CLP. Interestingly, CXCL16 is dispensable for controlling bacterial infection in CLP-induced polymicrobial sepsis. These results are consistent with a previous study showing that neutralization of CXCL16 resulted in reduced cerebrospinal 
fluid pleocytosis in an animal model of pneumococcal meningitis, but it did not influence bacterial outgrowth in the central nervous system irrespective of the time of administration [28]. In a murine model of collagen-induced arthritis (CIA), treatment with anti-CXCL16 monoclonal antibody has also been shown to significantly reduce the clinical arthritis score and reduce infiltration of inflammatory cells and bone destruction in the synovium of mice with CIA [9]. Neutralizing CXCL16 by injection of anti-CXCL16 antibody ameliorated the extent of hepatic inflammation and steatosis development in experimental metabolic injury [29]. Furthermore, administration of anti-CXCL16 antibody could ameliorate colonic inflammation in the experimental model of colitis [22]. In fact, sepsis was associated with a complex network of pro- and anti-inflammatory modulators, the balance of which determines the outcome of the sepsis. Taken together, sepsis was associated with elevated production of CXCL16, and CXCL16 had a limited antibacterial role in sepsis. However, CXCL16 could cause aberrant inflammatory response (increase of leukocyte infiltration including monocytes/macrophages, neutrophils and lymphocytes, and elevation of inflammatory cytokines/ chemokines including IL-6, TNF- $\alpha$, KC and IL-27), resulting in exaggerated inflammation, which can cause tissue injury and increased lethality. Therefore, targeting CXCL16 might therefore represent a promising novel therapeutic approach for the treatment of sepsis.

Some limitations of this study should be noted. First, this is a single-center study with a limited sample size, and our results should be validated in a larger ideally multicentric study. Second, the AUC of CXCL16 was lower than that of SOFA score in predicting 28-day mortality of septic patients, suggesting that incremental further studies are required to investigate whether the combination of CXCL16 with other risk biomarkers might provide additional prognostic value in septic patients. Finally, further investigation using CXCL16 knockout mice is required to elucidate the underlying molecular mechanisms by which CXCL16 amplifying local and systemic inflammation during sepsis.

Collectively, we here firstly establish that human septic patients had a stable and sustained elevation of soluble serum CXCL16 levels, which correlate with disease severity and mortality. Measuring admission levels of soluble serum CXCL16 could represent a promising biomarker for risk stratification and evaluation of prognosis in the patients with sepsis. In complementary experimental studies, administration with recombinant CXCL16 could aggravate nonsevere sepsis, while anti-CXCL16 treatment could ameliorate severe sepsis. Targeting the chemokine CXCL16 is a promising strategy in the treatment of sepsis. Taken together, CXCL16 emerges as a promising biomarker for disease severity and as a potential target for therapeutic intervention in human sepsis.

\section{Acknowledgments}

This work was supported by National Natural Science Foundation of China grants (No. 82070014 to JC and No. 81902134 to YG).

\section{Competing Interests}

The authors declare that there are no competing interests associated with the manuscript.

\section{REFERENCES}

1 Singer M, Deutschman CS, Seymour CW et al. The Third International Consensus Definitions for Sepsis and Septic Shock (Sepsis-3). JAMA 2016; 315: 801-10.

2 van der Poll T, van de Veerdonk FL, Scicluna BP et al. The immunopathology of sepsis and potential therapeutic targets. Nat Rev Immunol 2017; 17: 407-20.

3 Reinhart K, Daniels R, Kissoon N et al. Recognizing Sepsis as a Global Health Priority - A WHO Resolution. N Engl J Med 2017;377: 414-7.

4 Stolk RF, van der Pasch E, Naumann F et al. Norepinephrine Dysregulates the Immune Response and Compromises Host Defense during Sepsis. Am J Respir Crit Care Med 2020; 202: 830-42. 
5 Shimaoka T, Kume N, Minami M et al. Molecular cloning of a novel scavenger receptor for oxidized low density lipoprotein, SR-PSOX, on macrophages. J Biol Chem 2000; 275: 40663-6.

6 Chaparro V, Leroux LP, Zimmermann A et al. Leishmania donovani Lipophosphoglycan Increases Macrophage-Dependent Chemotaxis of CXCR6-Expressing Cells via CXCL16 Induction. Infect Immun 2019; 87: e00064-19.

7 Fahy OL, Townley SL, McColl SR et al. CXCL16 regulates cell-mediated immunity to Salmonella enterica serovar Enteritidis via promotion of gamma interferon production. Infect Immun 2006; 74: 6885-94.

8 Izquierdo MC, Martin-Cleary C, Fernandez-Fernandez B et al.CXCL16 in kidney and cardiovascular injury. Cytokine Growth Factor Rev 2014; 25: 317-25.

9 Nanki T, Shimaoka T, Hayashida K et al. Pathogenic role of the CXCL16-CXCR6 pathway in rheumatoid arthritis. Arthritis Rheum 2006;54: 765-78.

10 Hassan AM, Farghal NMA, Hegab DS et al. Serum-soluble CXCL16 in juvenile systemic lupus erythematosus: a promising predictor of disease severity and lupus nephritis. Clin Rheumatol 2018; 37:3025-32.

11 Ajona D, Zandueta C, Corrales L et al. Blockade of the Complement C5a/C5aR1 Axis Impairs Lung Cancer Bone Metastasis by CXCL16-mediated Effects. Am J Respir Crit Care Med 2018; 197:1164-76.

12 Kim MJ, Sun HJ, Song YS et al. CXCL16 positively correlated with M2-macrophage infiltration, enhanced angiogenesis, and poor prognosis in thyroid cancer. Sci Rep 2019; 9: 13288.

13 Gao Q, Zhao YJ, Wang XY et al. CXCR6 upregulation contributes to a proinflammatory tumor microenvironment that drives metastasis and poor patient outcomes in hepatocellular carcinoma. Cancer Res 2012;72: 3546-56.

$14 \mathrm{Tu}$ GW, Ju MJ, Zheng YJ et al. CXCL16/CXCR6 is involved in LPS-induced acute lung injury via P38 signalling. J Cell Mol Med2019; 23: 5380-9.

15 Landrø L, Damås JK, Halvorsen B et al. CXCL16 in HIV infection - a link between inflammation and viral replication. Eur J Clin Invest 2009; 39: 1017-24.

16 Lehrke M, Millington SC, Lefterova M et al. CXCL16 is a marker of inflammation, atherosclerosis, and acute coronary syndromes in humans. J Am Coll Cardiol 2007; 49: 442-9.

17 Smith C, Halvorsen B, Otterdal K et al. High levels and inflammatory effects of soluble CXC ligand 16 (CXCL16) in coronary artery disease: down-regulatory effects of statins. Cardiovasc Res 2008; 79: 195-203.

18 Andersen T, Ueland T, Ghukasyan Lakic T et al. C-X-C Ligand 16 Is an Independent Predictor of Cardiovascular Death and Morbidity in Acute Coronary Syndromes. Arterioscler Thromb Vasc Biol 2019;39: 2402-10.

19 Song Z, Zhang X, Zhang L et al. Progranulin Plays a Central Role in Host Defense during Sepsis by Promoting Macrophage Recruitment. Am J Respir Crit Care Med 2016; 194: 1219-32.

20 Lin X, Luo H, Yan X, et al . Interleukin-34 Ameliorates Survival and Bacterial Clearance in Polymicrobial Sepsis. Crit Care Med 2018; 46: e584-e90.

21 Yan X, Tu H, Liu Y et al. Interleukin-17D Aggravates Sepsis by Inhibiting Macrophage Phagocytosis. Crit Care Med 2020;48: e58-e65.

22 Uza N, Nakase H, Yamamoto S et al. SR-PSOX/CXCL16 plays a critical role in the progression of colonic inflammation. Gut 2011; 60: 1494-505.

23 Jansson AM, Aukrust P, Ueland T et al. Soluble CXCL16 predicts long-term mortality in acute coronary syndromes. Circulation 2009;119: 3181-8. 
24 Hoogerwerf JJ, Tanck MWT, Zoelen MAD et al. Soluble ST2 plasma concentrations predict mortality in severe sepsis. Intensive Care Med 2010; 36: 630-7.

25 Liu B, Chen YX, Yin Q et al. Diagnostic value and prognostic evaluation of Presepsin for sepsis in an emergency department. Crit Care 2013; 17:R244.

26 Demaret J, Villars-Méchin A, Lepape A et al. Elevated plasmatic level of soluble IL-7 receptor is associated with increased mortality in septic shock patients.Intensive Care Med 2014; 40: 1089-96.

27 Gao X, Liu Y, Xu F et al. Assessment of Apoptosis Inhibitor of Macrophage/CD5L as a Biomarker to Predict Mortality in the Critically Ill With Sepsis. Chest 2019; 156: 696-705.

28 Woehrl B, Klein M, Rupprecht TA et al. CXCL16 contributes to neutrophil recruitment to cerebrospinal fluid in pneumococcal meningitis. J Infect Dis 2010;202: 1389-96.

29 Wehr A, Baeck C, Ulmer F et al. Pharmacological inhibition of the chemokine CXCL16 diminishes liver macrophage infiltration and steatohepatitis in chronic hepatic injury. PLoS One 2014; 9:e112327.

A
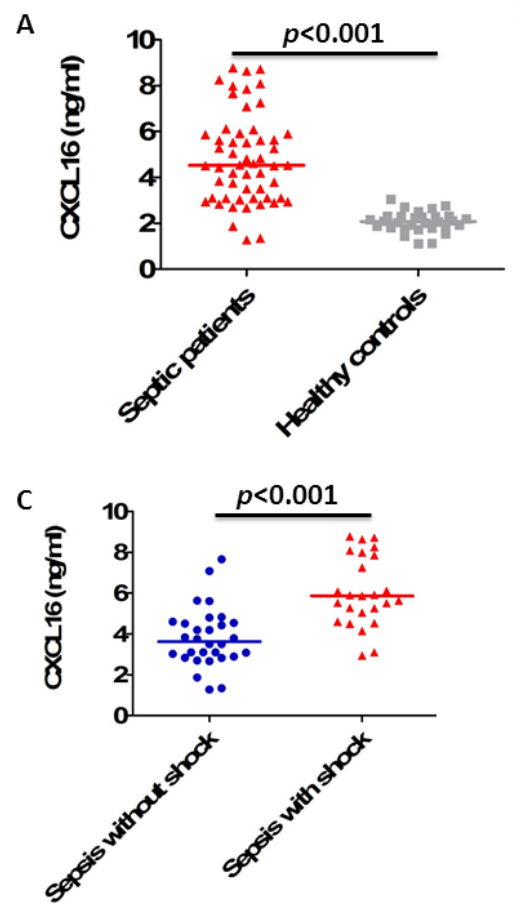

Figure 1

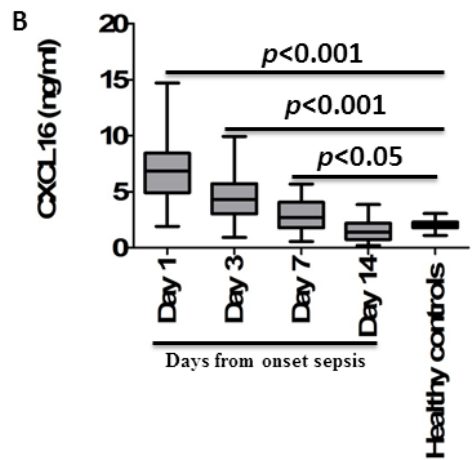

D

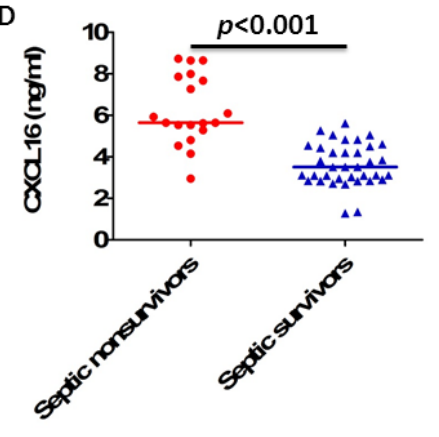

Figure 1. Circulating CXCL16 concentrations in the patients with sepsis were increased. (A) Soluble levels of serum CXCL16 in 55 septic patients and 30 healthy controls. (B) The dynamics of soluble CXCL16 levels in the serum of patients with sepsis. (C) Soluble CXCL16 levels in the serum collected from patients with septic shock and patients without shock on day of ICU admission. (D) Soluble CXCL16 levels in the serum collected from septic survivors and nonsurvivors. Nonparametric Mann-Whitney $U$ test or Kruskal-Wallis test followed by Dunn's multiple comparisons post test was performed to analyze results between groups. Horizontal bars represent median values, and dots represent individual participants. 

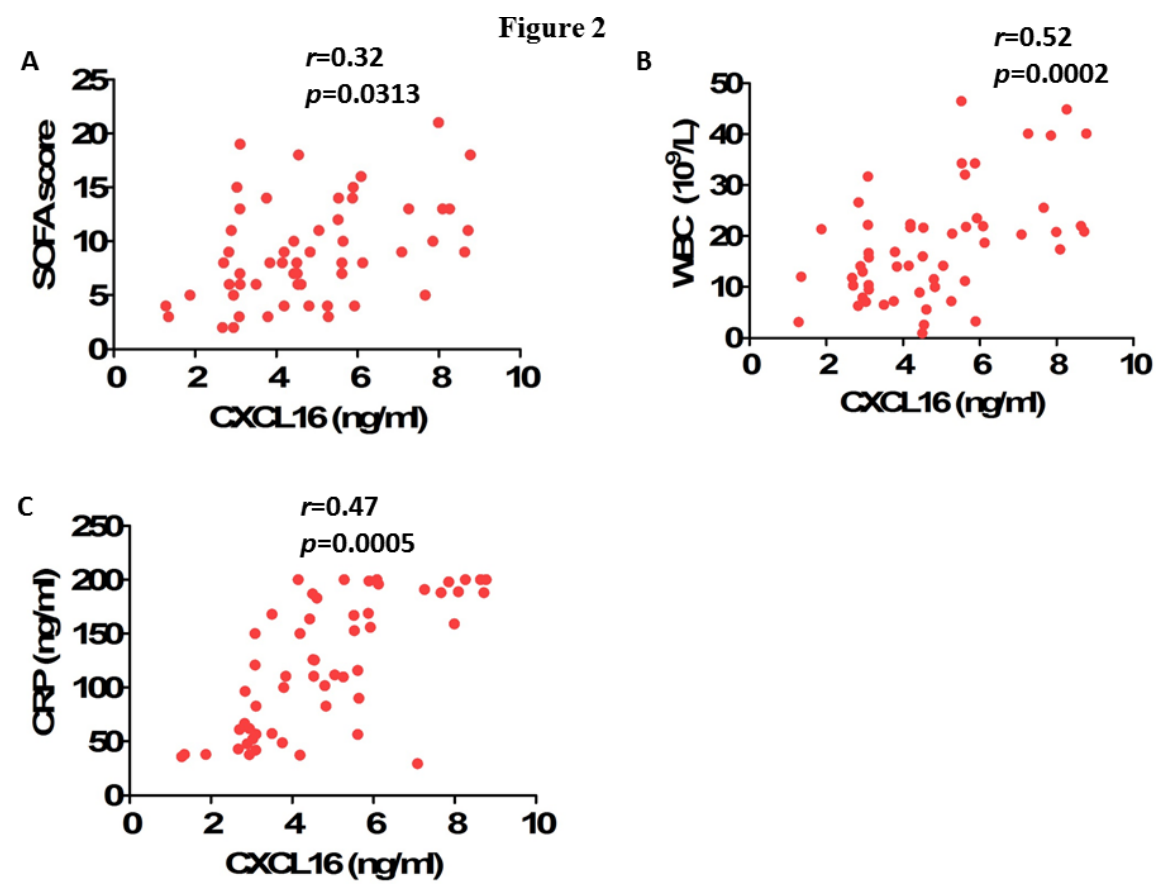

Figure 2. Soluble serum CXCL16 levels at admission correlated with disease severity in the patients with sepsis. (A) Correlation of soluble CXCL16 levels with Sequential Organ Failure Assessment (SOFA) scores in septic patients. (B) Correlation of soluble CXCL16 levels with white blood cells (WBC) in septic patients. (C) Correlation of soluble CXCL16 levels with C-reactive protein (CRP) levels in septic patients. Spearman's correlation coefficient was performed to analyze the correlation between the levels of CXCL16 and SOFA scores, WBC, or CRP levels. Horizontal bars represent median values, and dots represent individual participants. 


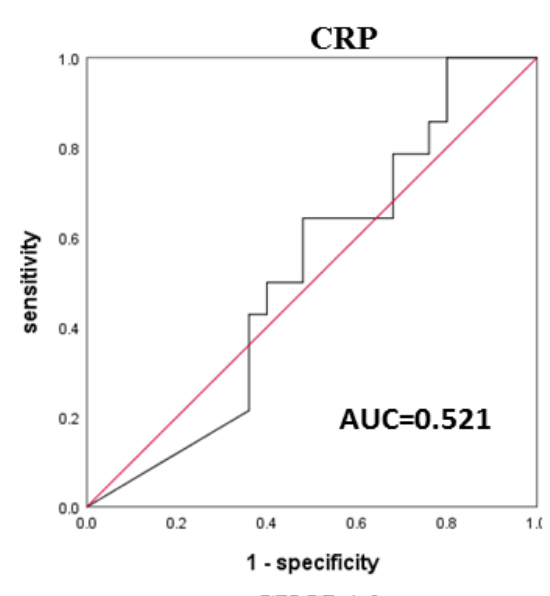

Figure 3

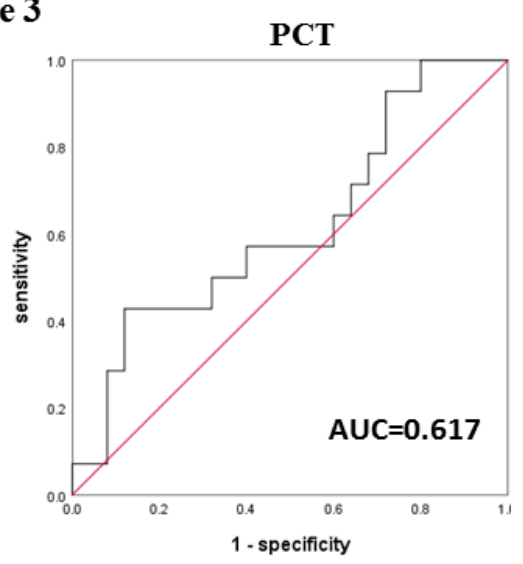

CXCL16
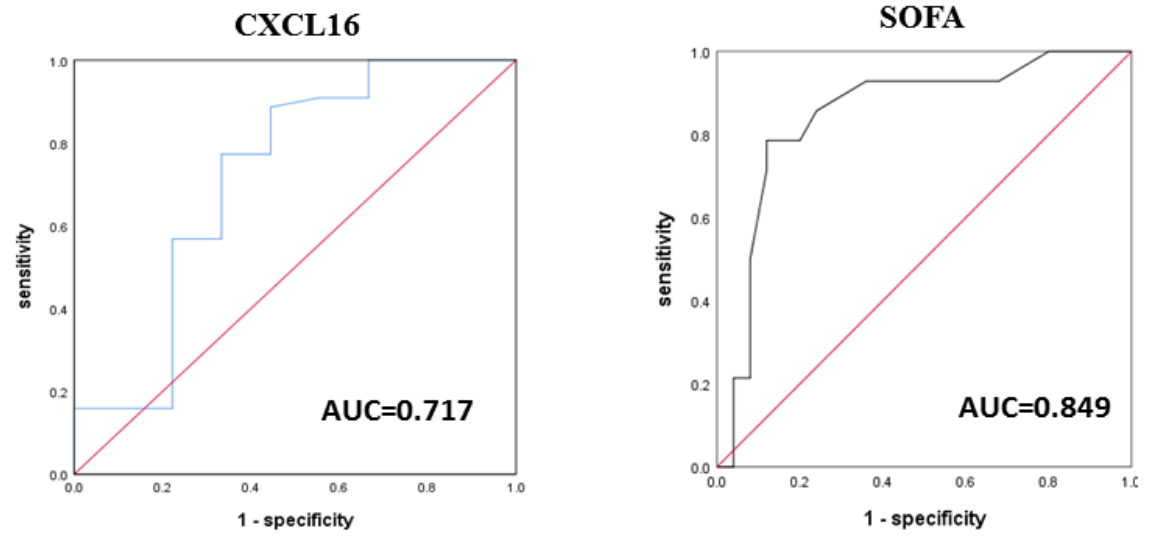

Figure 3 . Receiving operating characteristic (ROC) curve analysis of CXCL16, SOFA score, PCT, and CRP at admission for predicting 28-day mortality in septic patients. Area under the ROC curve: 0.717 ([95\% CI] 0.497-0.939) for CXCL16; 0.849 ([95\% CI] 0.715-0.982) for SOFA score; 0.617 ([95\% CI] 0.429-0.805) for PCT; and $0.521([95 \% \mathrm{CI}] 0.338-0.705)$ for CRP. 
A

Figure 4
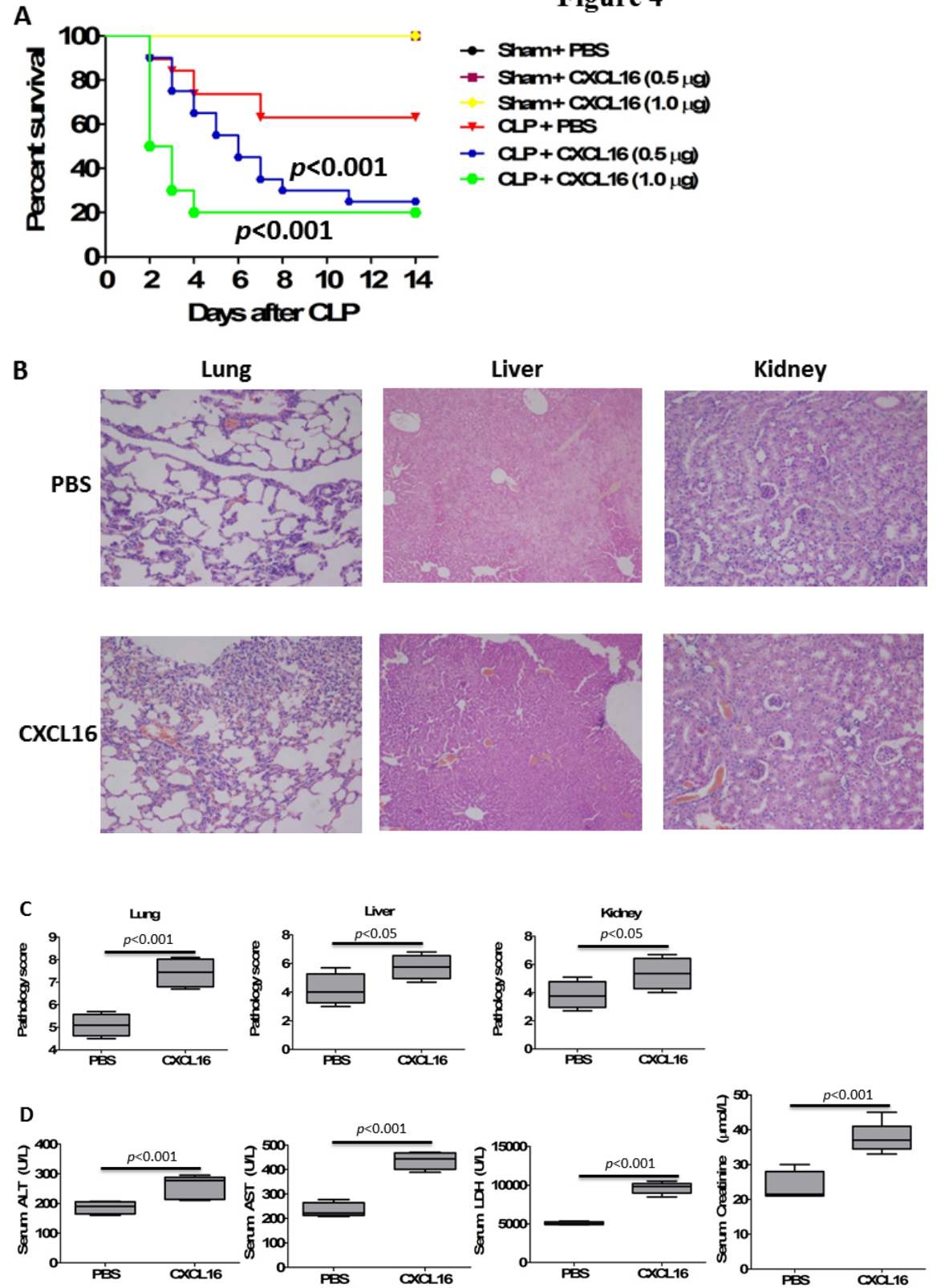

Figure 4. CXCL16 worsened cecal ligation and puncture (CLP)-induced nonsevere sepsis. (A) Survival of CLP mice ( $\mathrm{n}=12$ per group) after treatment with recombinant CXCL16 protein in the absence or presence of CLP-induced nonsevere sepsis. Murine recombinant CXCL16 protein was administered intraperitoneally at 0.5-1.0 $\mu \mathrm{g} /$ injection immediately after nonsevere CLP, and PBS was used as a control. Comparison between groups was performed using Kaplan-Meier analysis followed by log-rank tests. Results are representative of three independent experiments. $p<0.001$ when compared with septic mice treated with PBS control. (B) Representative examples of hematoxylin and eosin-stained lung, liver, and kidney tissues from nonsevere CLP mice $(\mathrm{n}=6)$ treated with or without recombinant CXCL16 $(0.5 \mu \mathrm{g} /$ injection). (C) Histological scores for lung, liver, and kidney in nonsevere CLP mice $(\mathrm{n}=6)$ treated with or without recombinant CXCL16 $(0.5 \mu \mathrm{g} /$ injection). $\quad p<0.05, p<0.001$ when compared with septic mice treated with PBS control (MannWhitney $U$ test). (D) ALT, AST, LDH, and creatinine levels in nonsevere CLP mice $(\mathrm{n}=6)$, treated with 
or without recombinant CXCL16 (0.5 $\mu \mathrm{g} /$ injection). $p<0.001$ when compared with septic mice treated with PBS control (Mann-Whitney $U$ test).

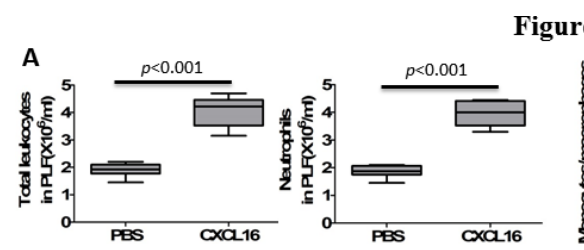

igure 5
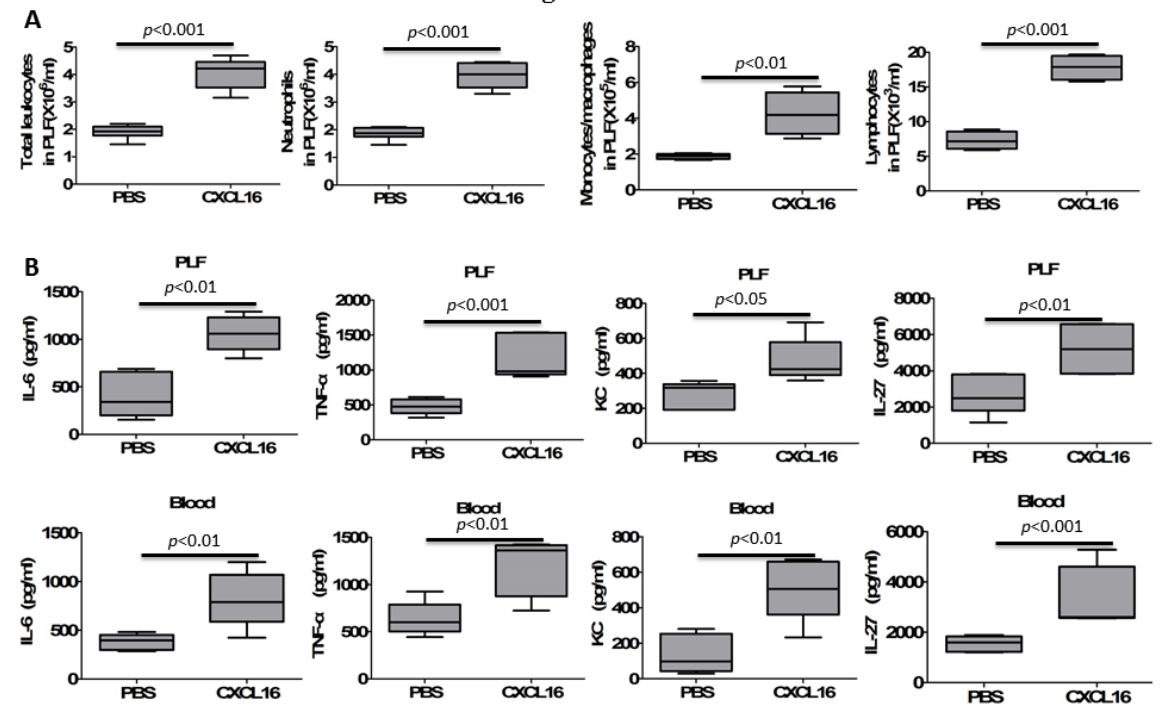

Figure 5 . Treatment with recombinant murine CXCL16 enhanced inflammatory response in CLP-induced nonsevere sepsis. (A) Numbers of leukocytes including neutrophils, monocytes/macrophages and lymphocytes in peritoneal lavage fluid (PLF) of septic mice treated with or without recombinant murine CXCL16 $(0.5 \mu \mathrm{g} /$ injection). (B) Cytokine and chemokine levels in PLF and blood from septic mice $(\mathrm{n}=5)$ treated with or without recombinant murine CXCL16 (0.5 $\mathrm{g}$ /injection) at $24 \mathrm{~h}$ after nonsevere CLP. $p<0.05, p$ $<0.01, p<0.001$ when compared with septic mice treated with PBS control (Mann-Whitney $U$ test). 
A

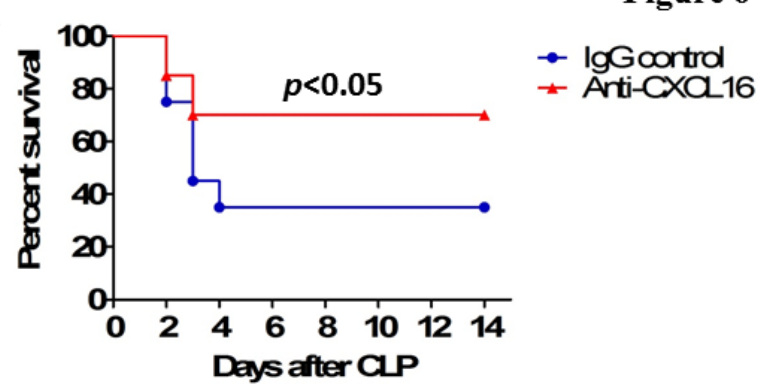

B
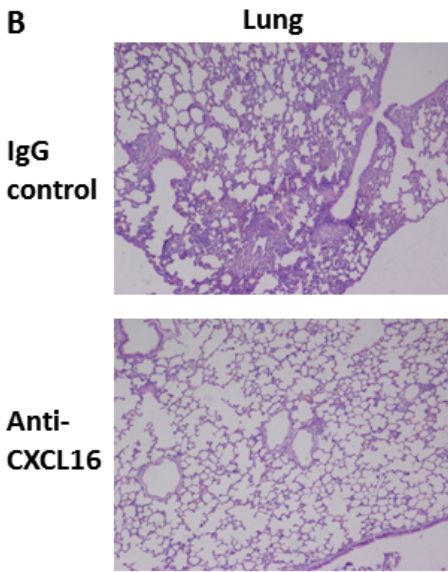

Liver
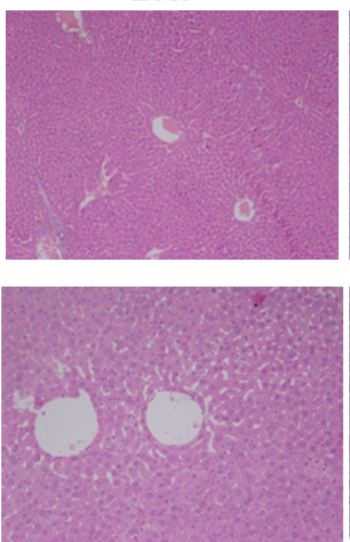

Kidney
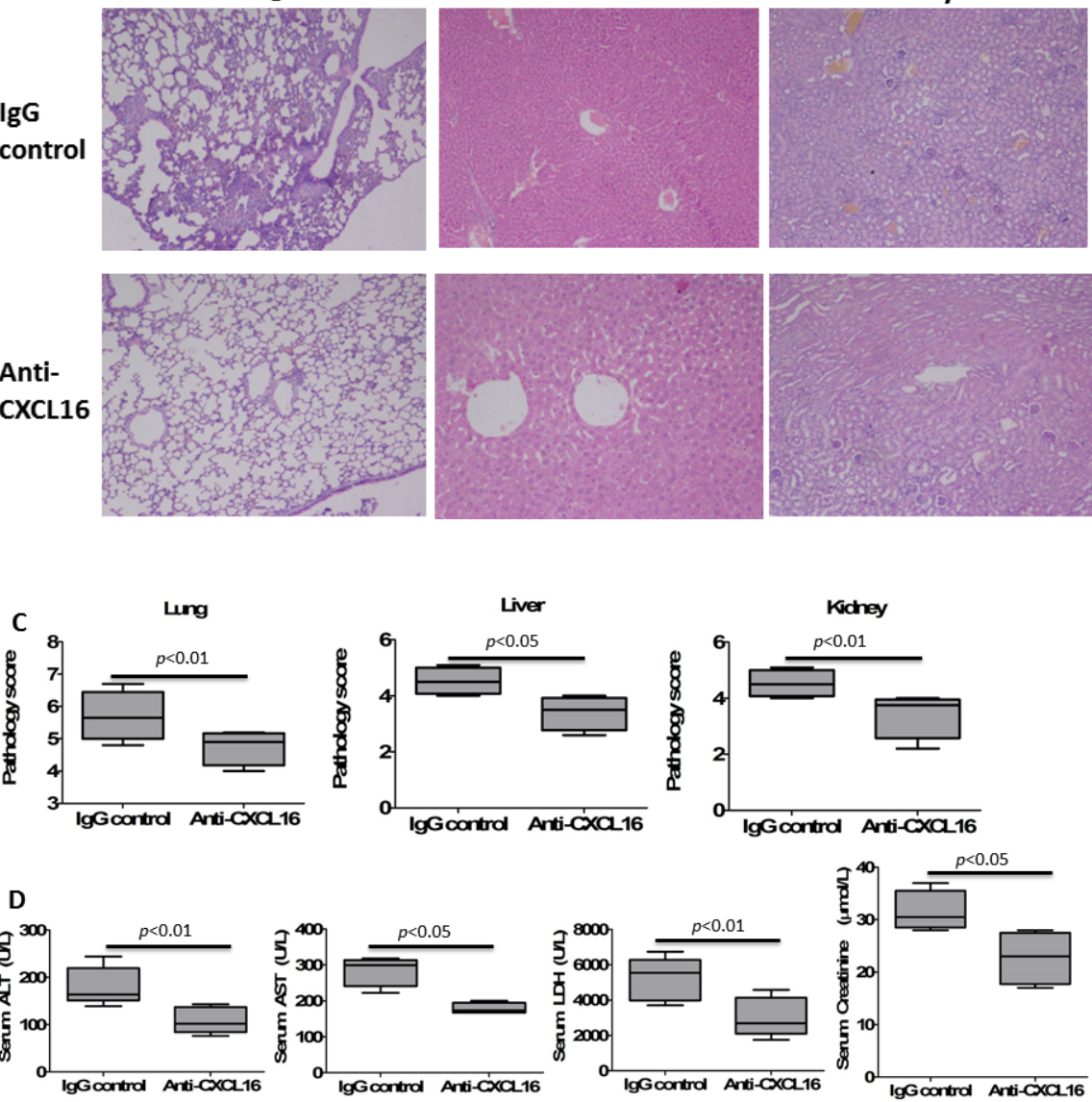

Figure 6 . Anti-CXCL16 antibody ameliorated cecal ligation and puncture (CLP)-induced severe sepsis. C57BL/6 mice were subjected to severe CLP; and then $10 \mu \mathrm{g}$ of anti-mouse CXCL16 monoclonal antibody was injected intraperitoneally at 2 hour after severe CLP. IgG2A control antibody was delivered in a similar way. (A) Survival of septic mice ( $\mathrm{n}=12$ per group) following administration with anti-CXCL16 antibody or control antibody after severe CLP. Comparison between groups was performed by Kaplan-Meier analysis followed by log-rank tests. Results are representative of three independent experiments. $p<0.05$ when compared with septic mice treated with $\operatorname{IgG}$ control. (B) Representative examples of hematoxylin and eosin-stained lung, liver, and kidney tissues from septic mice $(\mathrm{n}=6)$ treated with or without anti-CXCL16 neutralizing antibodies after severe CLP. (C) Histological scores for lung, liver, and kidney in septic mice $(\mathrm{n}=6)$ treated with or without anti-CXCL16 neutralizing antibodies after severe CLP. $p<0.05, p<0.01$ when compared with septic mice treated with isotypical IgG control (Mann-Whitney U test). (D) ALT, 
AST, LDH, and creatinine levels in septic mice $(\mathrm{n}=6)$ treated with or without anti- CXCL16 neutralizing antibodies after severe CLP. $p<0.05, p<0.01$ when compared with septic mice treated with isotypical IgG control (Mann-Whitney Utest).

Table 1. Independent factors predicting 28-day mortality in septic patients

Variable B Standard error Wald Degrees of freedom $P$ value Odds ratio $95 \%$ confidence interval

CXCL16 $0.0070 .0034 .01310 .0391 .0041 .000-1.010$

PCT $0.0130 .0063 .30310 .0741 .0110 .999-1.023$

CRP $0.0040 .0080 .85710 .3041 .0060 .983-1.033$

SOFA $0.0900 .02115 .61710 .0001 .0931 .050-1.140$

CRP: C-reactive protein; PCT: procalcitonin; SOFA: sequential organ failure assessment.

Supplementary Figure legends

Supplementary Figure 1. Admission levels of soluble CXCL16 in serum samples from septic patients without bacteremia and with documented bacteremia

Supplementary Figure 2. CXCL16 production was elevated in septic mice after cecal ligation puncture (CLP). C57BL/6 mice ( $\mathrm{n}=5$ per group) were subjected to sham or nonsevere CLP with a 24-gauge needle, and then samples were collected for CXCL16 quantification by ELISA. $p<0.001$ when compared with sham control mice (Mann-Whitney $U$ test).

Supplementary Figure 3. The effects of recombinant murine CXCL16 on bacterial clearance in cecal ligation and puncture (CLP)-induced nonsevere sepsis. Dilutions of peritoneal lavage fluid (PLF) and blood were collected from CLP mice $(n=6)$ administered with or without recombinant murine CXCL16 (0.5 $\mu \mathrm{g}$ ), and then were cultured on blood agar plates, and the number of bacterial colonies was determined as colony-forming unit (CFU).

Supplementary Figure 4. Administration with anti-CXCL16 antibody did not affect bacterial clearance in CLP-induced severe sepsis. Dilutions of peritoneal lavage fluid (PLF) and blood were collected from CLP mice $(\mathrm{n}=6)$ treated with or without anti-CXCL16 antibody $(10 \mu \mathrm{g})$, and then were cultured on blood agar plates, and the number of bacterial colonies was determined as colony-forming unit (CFU).

Supplementary Table 1 . Characteristics of septic patients and healthy controls

Supplementary Table 2. Pathogens responsible for infections in the patients with sepsis

Supplementary Table 3. Baseline characteristics of septic survivors and non-survivors in the patients with sepsis

Supplementary Table 4. Correlations between CXCL16 and other cytokine levels in the patients with sepsis 